\title{
Power quality event classification: an overview and key issues
}

\author{
D. Saxena" ${ }^{* 1}$, K.S. Verma" ${ }^{\#}$ and S.N. Singh ${ }^{+}$ \\ * Department of Electrical and Electronics Engineering, Invertis Inst. of Engg.\& Tech., Bareilly (UP), INDIA. \\ ${ }^{+}$Department of Electrical Engineering, CET, Denmark Technical University, Kgs. Lyngby, DENMARK. \\ ${ }^{\#}$ Department of Electrical Engineering, K.N.I.T Sultanpur (UP), INDIA. \\ E-mails: diptisx@gmail.com (D. Saxena ${ }^{1},{ }^{1}$ Corresponding author), snsingh@iitk.ac.in (S.N. Singh), ksv02@rediffmail.com (K.S. Verma)
}

\begin{abstract}
Power quality (PQ) issue has attained considerable attention in the last decade due to large penetration of power electronics based loads and/or microprocessor based controlled loads. On one hand these devices introduce power quality problem and on other hand these mal-operate due to the induced power quality problems. PQ disturbances/events cover a broad frequency range with significantly different magnitude variations and can be non-stationary, thus, accurate techniques are required to identify and classify these events/disturbances. This paper presents a comprehensive overview of different techniques used for PQ events' classifications. Various artificial intelligent techniques which are used in PQ event classification are also discussed. Major Key issues and challenges in classifying PQ events are critically examined and outlined.
\end{abstract}

Keywords: Power quality, PQ event classifiers, artificial intelligence techniques, PQ noise, PQ key issues.

\section{Introduction}

An electrical power system is expected to deliver undistorted sinusoidal rated voltage and current continuously at rated frequency to the end users. However, large penetration of power electronics based controllers and devices along with restructuring of the electric power industry and small-scale distributed generation have put more stringent demand on the quality of electric power supplied to the customers (Arrillaga et al, 2000a; Arrillaga et al, 2000b; Dugan et al, 2003). To define power quality (PQ), the views of utilities, equipment manufacturers, and customers are completely different. Utilities treat PQ from the system reliability point of view. Equipment manufacturers, on the other hand, consider PQ as being that level of power supply allowing for proper operation of their equipment, whereas customers consider good PQ that ensures the continuous running of processes, operations, and business. A PQ problem can be defined as "any power problem manifested in voltage, current and/or frequency deviations that result in failure or mal-operation of customers' equipment”. In early days, power quality (Martzloff et al, 1998) issues were concerned with the power system transient arising due to switching and lightning surges, induction furnace and other cyclic loads. Increased interconnection, widespread use of power electronics devices with sensitive and fast control schemes in electrical power networks have brought many technical and economic advantages, but these have also introduced new challenges for the power engineers (Burke et al, 1990; Domijan et al, 1993).

In new electricity market scenarios, now electricity consumers can shift to the new service providers, if power quality is not good. Moreover, these customers can demand a higher quality of service. The utilities or other electric power providers have to ensure a high quality of their service to remain competitive and to retain/attract the customers. The power quality term was earliest mentioned in a study published in 1968 (Kajihara et al, 1968) following many publications (Martzloff et al, 1998) mentioned PQ as voltage quality with reference to slow variation in voltage magnitude. The power quality analysis was, first, started at the end of $19^{\text {th }}$ century, when rotating machinery and transformers were found to be main sources of the waveform distortion (Acha et al, 2001). PQ problems fall into two basic categories (Dwivedi et al, 2008):

\footnotetext{
${ }^{1}$ Corresponding author. Tel: +91- 9410261368
} 
1. Events or Disturbances: Disturbances are measured by triggering on an abnormality in the voltage or the current. Transient voltages may be detected when the peak magnitude exceeds a specified threshold. RMS voltage variations (e.g., sags or interruptions) may be detected when it exceeds a specified level.

2. Steady-State Variations: Steady state variation is basically a measure of the magnitude by which the voltage or current may vary from the nominal value, plus distortion and the degree of unbalance between the three phases. These include normal rms voltage variations, and harmonic and distortion.

The power quality events/disturbances can further be classified according to the nature of the waveform distortion. The information regarding typical spectral content, duration and magnitude for each category of electromagnetic disturbances are shown in Table 1. The phenomena listed in the Table 1 can be described further by various appropriate attributes. For steady-state disturbances, the amplitude, frequency, spectrum, modulation, source impedance, notch depth and notch area attributes can be utilized. However, for non-steady state disturbances, other attributes such as rate of rise, rate of occurrence and energy potential are useful.

The main reasons for the increased interest in PQ can be summarized as follows:

- Modern electric appliances are equipped with power electronics devices utilizing microprocessor/microcontroller. These appliances introduce various types of PQ problems and moreover, these are very sensitive to the PQ problems.

- Industrial equipments such as high-efficiency, adjustable speed motor drives and shunt capacitors are now extensively used. The complexity of industrial processes, which results in huge economic losses if equipment fails or malfunctions.

- The complex interconnection of systems, resulting in more severe consequences if any one component fails. Moreover, various sophisticated power electronics equipments, which are very sensitive to the PQ problems, are used for improving system stability, operation and efficiency.

- There has been a significant increase in embedded generation and renewable energy sources which create new power quality problems, such as voltage variations, flicker and waveform distortions.

- Introduction of competitive electricity market gives right to the customers to demand high quality of supply.

Table 1: Classification of various power quality events

\begin{tabular}{|c|c|c|c|}
\hline S. No. & Categories & Duration & Voltage Magnitude \\
\hline I & $\begin{array}{ll}\text { Short Duration } & \text { Variation } \\
\text { Sag } & \text { Instantaneous } \\
& \text { Momentary } \\
& \text { Temporary } \\
\text { Swell } & \text { Instantaneous } \\
& \text { Momentary } \\
& \text { Temporary } \\
\text { Interruption } & \text { Momentary } \\
& \text { Temporary }\end{array}$ & $\begin{array}{r}0.5-30 \text { cycle. } \\
30 \text { cycles-3 sec. } \\
3 \text { sec- } 1 \mathrm{~min} . \\
0.5-30 \text { cycle. } \\
30 \text { cycles-3 sec. } \\
\text { 3sec- } 1 \mathrm{~min} . \\
0.5 \text { cycles-3sec. } \\
\text { 3sec-1min. }\end{array}$ & $\begin{array}{r}0.1-0.9 \text { pu. } \\
0.1-0.9 \text { pu. } \\
0.1-0.9 \text { pu. } \\
1.1-1.8 \mathrm{pu} . \\
1.1-1.4 \mathrm{pu} . \\
1.1-1.2 \mathrm{pu} . \\
<0.1 \mathrm{pu} . \\
<0.1 \mathrm{pu} .\end{array}$ \\
\hline $\begin{array}{l}\text { II } \\
\text { (a) } \\
\text { (b) } \\
\text { (c) }\end{array}$ & $\begin{array}{l}\text { Long Duration Variation } \\
\text { Interruption, Sustained } \\
\text { Under-voltage } \\
\text { Overvoltage }\end{array}$ & $\begin{array}{l}>1 \text { min } \\
>1 \text { min } \\
>1 \text { min }\end{array}$ & $\begin{array}{r}0.0 \mathrm{pu} . \\
0.8-0.9 \mathrm{pu} . \\
1.1-1.2 \mathrm{pu}\end{array}$ \\
\hline III (a) & $\begin{array}{ll}\text { Transients } & \\
\text { Impulsive } & \text { Nanosecond } \\
& \text { Microsecond } \\
& \text { Millisecond } \\
\text { Oscillatory } & \text { Low frequency } \\
& \text { Medium freq. } \\
& \text { High freq. }\end{array}$ & $\begin{array}{r}<50 \mathrm{nsec} . \\
50-1 \mathrm{msec} . \\
>1 \mathrm{msec} . \\
0.3-50 \mathrm{msec} . \\
20 \mu \mathrm{sec} . \\
5 \mu \mathrm{sec} .\end{array}$ & $\begin{array}{l}0-4 \text { pu. } \\
0-8 \mathrm{pu} . \\
0-4 \mathrm{pu} .\end{array}$ \\
\hline IV & Voltage Imbalance & Steady state & $0.5-2 \%$ \\
\hline $\begin{array}{l}\mathrm{V} \\
\text { (a) } \\
\text { (b) } \\
\text { (c) }\end{array}$ & $\begin{array}{l}\text { Waveform Distortion } \\
\text { Harmonics } \\
\text { Notching } \\
\text { Noise }\end{array}$ & $\begin{array}{l}\text { Steady state } \\
\text { Steady state } \\
\text { Steady state }\end{array}$ & \\
\hline
\end{tabular}

To minimize power quality disturbances and to devise suitable corrective and preventive measures, efficient detection and classification techniques are required in the emerging power systems. Classification of power quality disturbances based on the 
visual inspection of waveforms by human operators is laborious and time consuming. Moreover, it is not always possible to extract important information from simple visual inspection. The classification of PQ disturbances in electric power systems has become an important task for proper developing and designing the preventive and corrective measures.

Extensive research work and books are now available in the area of power quality. In this paper, a comprehensive survey of different classifications techniques used for PQ events has been presented. Various artificial intelligent techniques which are used in PQ event classification are also discussed. Major key issues and challenges in classifying PQ events are critically analyzed and presented.

\section{Automatic Power Quality Classifiers}

A basic block diagram of automatic classifier is shown in Fig. 1. The disturbance signal is passed to the pre-processing unit having two function blocks: segmentation and feature extraction. Extracted features are used to classify the PQ events. The classifier's information is used to make the final decision through the post-processing unit. The various blocks of automatic classification system is described below.

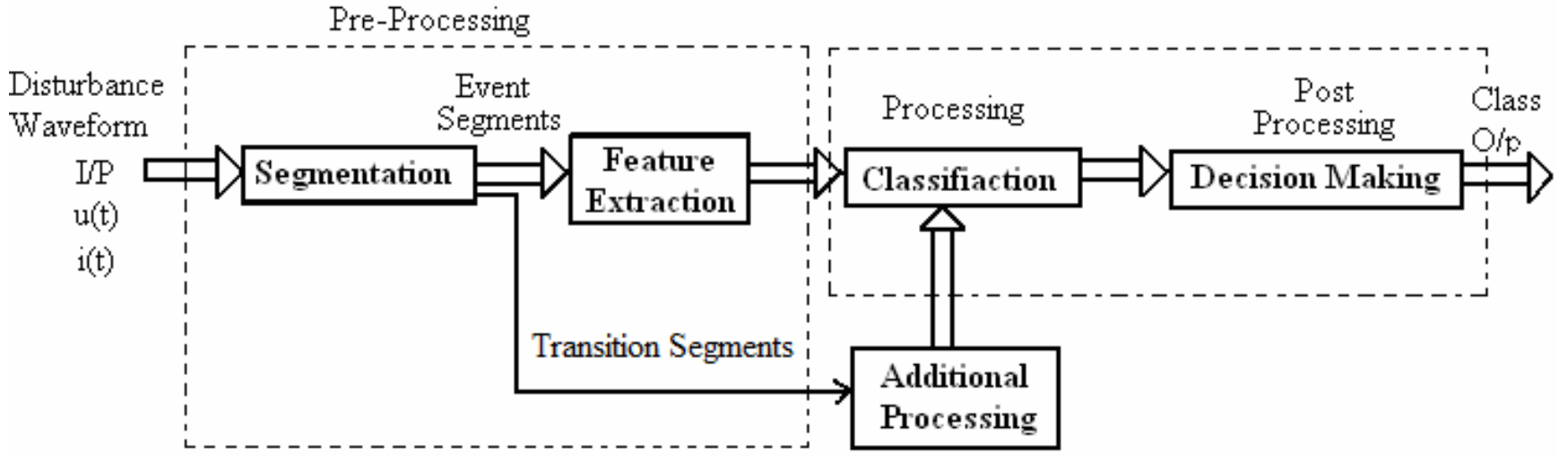

Figure 1. Block diagram of automatic power quality classifier

\subsection{Segmentation}

It is a preprocessing technique which divides the data sequence into stationary and non-stationary parts i.e. data is divided into a large number of transition segments (corresponding to large and sudden change in signal, and event segments). Events are the segments in between transition segments. The features are extracted from the event segments because the signal is stationary in this segment and normally contains the information that is unique enough to distinguish between different types of disturbances. To capture disturbance waveform period, a triggering method is required to get start and end time instant of PQ event (Bollen et al, 2006).

The current methods used for detecting PQ disturbances are based on a point-to-point comparison of adjacent cycle or a pointto point comparison of the RMS values of the distorted signal with its corresponding pure signal and/or frequency domain transformed data. The recent methods proposed for this purpose are parametric (or model based) such as Kalman filter (KF) and auto-regressive (AR) models, and nonparametric (or transform based) such as short-term Fourier transform (STFT) and wavelet transform (WT). Parametric methods exploit the prominent residuals obtained by fitting the captured waveform into the chosen model and nonparametric methods by finding singular points from multi-state decomposition of PQ signal. Residual signal from a model can be used for detecting transition points and for analyzing and characterizing the disturbances. The basic idea in KF and AR residuals based detection and triggering method is to fit the PQ data into a chosen KF or AR model.

\subsection{Features Extraction}

The selection of suitable features is extremely important for classification of any problem. The features extracted by signal processing techniques are used as input to the PQ classification system. Feature extraction can be defined as transformation of the raw signal from its original form to a new form, from which suitable information can be extracted. An appropriately chosen feature set reduces the burden over the classifiers. Feature may directly be extracted from the original measurements (e.g., RMS values), or from some transformed domain (e.g., Fourier, wavelet, STFT, and S-transform) or from the parameters of signal models (e.g., sinusoid KF and AR models).

Fourier transform (FT) has been used for extracting the frequency contents of the recorded signal. According to the frequency contents of the signal, some of the PQ problems can be detected (Allen et al, 1977; Altes et al, 1980). But, FT is not suitable for non-stationary signals. This is because FT provides information only about the existence of a certain frequency component, but 
does not give information about component appearance time. A suitable way to obtain such information is to apply time-frequency (or time scale) signal decomposition where time-evolved signal components in different frequency bands can be obtained. Although, STFT can partly alleviate this problem, but STFT still has the limitation of a fixed window width i.e. the trade-off between the frequency resolution and the time resolution should be determined a priori to observe a particular characteristic of the signals (Gu et al, 2000). Due to a fixed window width, STFT is inadequate for the analysis of the transient non-stationary signals. Therefore, more powerful and efficient methods and techniques are required to detect and analyze non-stationary disturbances.

To resolve the fixed resolution problem of STFT, many researchers have proposed the use of the WT approach to analyzing the power system disturbances (Santoso et al, 1996; Perunicic et al,1998; Santoso et al, 2000a; Ibrahim et al, 2002; Zhu et al, 2004; Abdel-Galil et al, 2004). The WT approach prepares a window that automatically adjusts to give proper resolutions of both the time and the frequency. In this approach, a larger resolution of time is provided to high-frequency components of a signal, and a larger resolution of frequency to low-frequency components. These features make the WT well suited for the analysis of the power system transients caused by various PQ disturbances.

S-transform has been also introduced recently in (Dash et al, 2003) as a new PQ signal analysis and feature extraction tool. In (Chilukuri et al, 2004), the features of seven simulated signals have been extracted by calculating the minima and maxima of the S-transform absolute matrix. Similar to the STFT, S-transform also requires significant amount of computational resources. This is due to the fact that the S-transform matrix is calculated by performing the inverse.

\subsection{PQ Classifier}

Automatic classifiers used for classification of different power quality disturbances can be broadly classified into deterministic and statistical classifiers as described below:

- Deterministic Classifiers: These classifiers can be designed with limited amount of data with sufficient power system expert knowledge. Rule based expert system and fuzzy expert system come under deterministic classification method.

- Statistical Classifiers: Statistical methods are suitable when a large amount of data from training of the classifiers is available. Artificial neural network (ANN) and support vector machines (SVM) are the statistical based classifiers. The artificial neural network is more popular in literature and often combined with set of wavelet filters for feature extraction and fuzzy logic for decision making.

\subsection{Decision-Making}

In most of the classifiers, this stage is merged with the classification stage. It assigns the type event to the current event. There should be a proper decision tool to increase the accuracy of classification. Normally, expert system and fuzzy logic are used as decision making tools.

\section{Classification Approaches}

Both conventional and artificial intelligence (AI) based classification methods are reported in the literature. The limitations of conventional methods are overcome by the AI based methods. Some frequently used AI based classifiers are rule-based expert systems, fuzzy classification systems, artificial neural networks, kernel machines, and support vector machines. The artificial neural network is the most popular method in literature often combined with other AI techniques. Reference (Bollen et al, 2007) has shown the advantage of statistical classifiers over the expert system based approach. Various classifiers are discussed in the subsections given below.

\subsection{Artificial Neural Network Based Classifiers}

Neural network is a non-linear, data driven self adaptive method and is a promising tool for classification. These can adjust themselves to the data without any explicit specification of functional or distributional form for the underlying model (Santoso et al, 2000c; Bishop, 1995). The neural network recognizes a given pattern by experience which is acquired during the learning or training phase when a set of finite examples is presented to the network. This set of finite examples is called the training set, and it consists of input patterns (i.e., input vector) along with their label of classes (i.e., output). In this phase, neurons in the network adjust their weight vectors according to certain learning rules. After the training process is completed, the knowledge needed to recognize patterns is stored in the neurons' weight vectors. The network is, then, presented to another set of finite examples, i.e., the testing data set, to assess how well the network performs the recognition tasks. This process is known as testing or generalization. ANN is a universal function approximator i.e. this can approximate any function with arbitrary accuracy. All the above mentioned attributes make ANN flexible in modeling real world complex problems.

Santoso et al (2000b) have classified six types of PQ events using wavelets and multiple neural networks. The classifier uses wavelet transform coefficient at five-scale signal decomposition level as input to multiple neural. The squared wavelet transform coefficients (SWTC) at each scale are used as inputs to the multiple neural networks for classifying the disturbances type. The 
architecture of the network is learning vector quantization (LVQ). The final decision for the disturbances type is made by combining the outcomes of multiple neural networks by using two- decision making schemes. One is simple voting scheme and the other is Dempster-Shafer theory of evidence. The proposed classifier is able to provide a degree of belief for the identified waveform. The classifier is able to achieve the accuracy rate of $90 \%$ by rejecting less than $10 \%$ of the waveform as ambiguous. The scheme is not good for sag and monitoring interruptions because of the fact that the irregularities caused by these disturbances are not significantly irregular compared to $60 \mathrm{~Hz}$ background sinusoidal signal and the time scale over which they occur is large.

Discrete wavelet transform (DWT) has been widely used to capture the transient occurrence and exact frequency features of PQ disturbances. DWT technology has been integrated with AI technologies to recognize and classify the PQ disturbances accurately. It was demonstrated by (Mo et al, 1997) how to extract features from wavelet transform coefficients at different scales that can be applied as input to neural networks for classification of non stationary signal type. Angrisani et al (1998) proposed to employ disturbances time duration estimated by continuous wavelet transform (CWT) and disturbances amplitude estimated by DWT to classify transient disturbance type. To extract the squared wavelet transform coefficients at each scale as inputs to neural networks for classification of the disturbances have been proposed in (Santoso et al, 1996; Santoso et al, 2000c; Gaouda et al, 1999). (Perunicic et al, 1998) used DWT coefficient as inputs to a single layer self organizing map neural network to train and classify the transient disturbance type. An effective wavelet multi-resolution single decomposition method was proposed for analyzing the power quality transient events based on standard derivation (Gaouda et al, 1999) and root mean square value (Gaouda et al, 2002a).

Although wavelet transform emerges as a powerful tool for PQ analysis, employing DWT coefficients directly as inputs to the neural networks require large memory space and much learning time. The computing efficiency can be enhanced if decomposition levels with the number of extraction features can be reduced. (Giang, 2004) proposed a classifier integrating discrete wavelet transform (DWT) with probabilistic neural network (PNN). The energy distribution features of distorted signal at 13 resolution levels are extracted using the wavelet multi-resolution analysis (MRA) technique and the Parseval's theorem. It has been found that energy values of decomposed signal at different level are sensitive to the type of disturbances. With the help of Parseval's Theorem, the quantity of extracted features of distorted signal is reduced without losing its property and hence requirement of memory space and computing time for proper classification of disturbance types is less. PNN is one of the supervised learning networks and it differs from other network in learning process (Specht, 1990). There is no requirement to set the initial weights of the network and no learning is required.

He et al (2006) presented the classification of seven types of PQ events based on wavelet transform and self organizing learning array (SOLAR) system. Wavelet MRA is used to calculate energy at each decomposition level which serves as input variable for SOLAR classification which has three advantages over a typical neural network: data driven learning, local interconnection and entropy based self organization. The proposed classifier has a robust anti-noise performance and it can achieve high overall classification accuracy even when the signal-to-noise ratio (SNR) is very low. Comparison with support vector machine (SVM) and inductive inference approach of classification (Abdel-Galil et al, 2004), SOLAR based on wavelet feature extraction can effectively classify PQ disturbances.

Though WT exhibits notable capabilities for detection and localization of disturbances, however, its compatibilities are often degraded due to the presence of noise mixed with measured waveform (Dwivedi et al, 2008; Santoso et al, 2000a; Santoso et al, 1996; Gaouda et al, 1999; Lee et al, 1997; Lin et al, 1998; Yang et al, 2001; McGranaghan et al, 2007). In particular when the spectrum of noise coincides with that of transient signals, the effects of the noise cannot be excluded by the filters without affecting the performance of these advanced digital signal processing (DSP) based method.

It has been shown that the use of S-transform for features extraction with neural network classifier led to a very high degree of classification for signals contaminated with $40 \mathrm{~dB}$ noise (almost pure signals). However, the accuracy has been dropped to $75 \%$ if contaminated noise strength has increased. Mishra et al (2008) proposed an S-transform based probabilistic neural network (PNN) classifier for classification of 11 types PQ disturbances with only four extracted features. Integrating S-transform with PNN can effetely detect and classify PQ disturbances even under noisy condition. Comparison of PNN with other two well known neural networks i.e., feed forward multilayer back propagation (FFML-BP) and learning vector quantization (LVQ) shows that PNN classifies events more effectively than FFML and LVQ.

The main drawbacks of ANN for PQ classifications are

- There is no rule to determine the data required for the training of each disturbance types. More data available for training will give better accuracy but will increase time to train the network. A classifier trained with a small data set is very accurate in identifying the training data set, but it is potentially unable to identify other data sets.

- The generalization performance of ANN is not guaranteed and could be poor on selection of training data. The performance is heavily dependent on the selection of training data set and the structure (or topologies) of neural networks (e.g. the number of hidden layers, neurons, the interconnection of sub neural networks if employed).

K-nearest neighbor $(\mathrm{kNN})$ pattern recognition technique has been applied to classify the PQ disturbances in (Gaouda et al, 2001; Gargoom et al, 2007). The kNN technique requires a large capacity of memory to store the training data. In (Gaouda et al, 2002b), three pattern recognition techniques (minimum Euclidean distance, kNN, and neural network) have been proposed to automatically classify PQ disturbances. The difference in energy distribution between the distorted power signal and the pure one, 
and the measured duration of the distortion event have been used to form feature vector for the proposed pattern recognition techniques. It was found that the error rate for neural network classifier is generally better than that for the other two pattern recognition techniques. The NN classifier shows higher overall performance even with high noise levels. The NN classifier is the least sensitive to noise level variation in the input data.

\subsection{Expert System Based Classifier}

The main drawback of ANN technique is the need of training cycle and requirement of retraining the entire ANN for every new PQ event (Chung et al, 2002; Youssef et al, 2004). In (Chung et al, 2002), first, a rule-based method has been used to classify time-characterized disturbances, and then, a wavelet method has been utilized to obtain a more flexible time frequency information. A hidden Markov model has also been adopted to determine the disturbance existence. A scalable event identification system (a rule based expert system) has been introduced in (Santoso et al, 2000b) to present an alternative approach to ANN in identifying power quality events. However, its poor learning ability and adaptability limit its application in real practice. Knowledge of power quality experts plays an important role in identification of power quality disturbances. Knowledge from power quality experts can be implemented in automatic classification system by developing a set of classification rules and implementing rules in a expert system (Grenier 1983; Agostino et al, 1986; Ashton et al, 1990; Domijan et al, 1993; Shapiro et al, 1993). These expert system consists of a set of rules where the "real intelligence" by human experts is translated into artificial intelligence" for computers. The expert system consists of three basic elements (Hayes-Roth et al, 1983; Gevarter, 1985) as shown in Fig. 2.

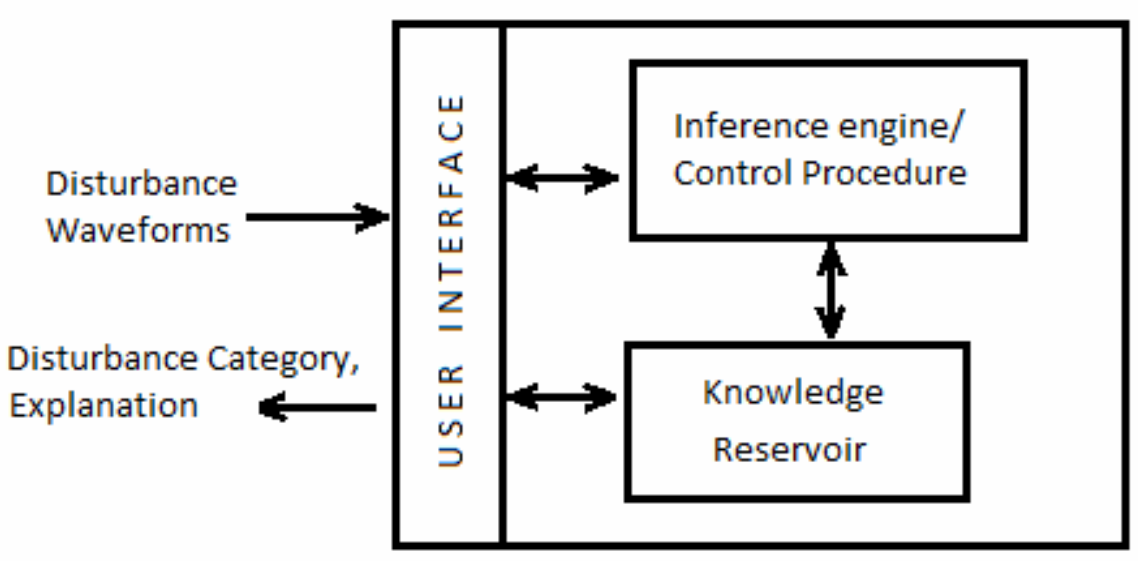

Figure 2. Basic structure of an expert system

The first element is the inference engine or the control procedure mechanism. This element draws inferences based on previously available knowledge and controls the flow of analysis. The second element is the knowledge reservoir, which is a collection of static knowledge represented by production rules (or if then rules). The third element is a user interface which facilitates the communication between users and the expert system. The structure of the expert system described above is indeed a common structure found in many expert system literatures.

The expert system identifies the PQ event by comparing the extracted feature from a given waveform from the knowledge stored in the system knowledge base by rule base of technique which consists of set of production rules. The expert systems are scalable i.e. they can be easily expanded for any new PQ event identification merely by just adding a new knowledge base module for the new PQ event, whereas in trained ANN it is difficult. If the expert system misclassifies, the associated production rules can always be debugged. However, it is not possible in ANN based classifiers without modifying the structure, the number of neuron or training strategy. In (Styvaktakis et al, 2001), expert systems have been used for classification of voltage dips. Reference (Styvaktakis et al, 2002) has also proposed an experts system for the classification and analysis of a number of power system events. Beside, few more papers (Kazibwe et al, 1992; Schlabbach 1994; Kezunovic et al, 1999; Reaz et al, 2007) have used the expert system as the PQ classifier. As the number of events or features increases, the complexity of the expert systems also increases. When data are abundant and it is difficult for human experts to characterize the given PQ event, ANN classifiers are preferred.

\subsection{Fuzzy Expert System Based Classifiers}

Fuzzy logic with rule based expert system has emerged as a powerful categorization tool for PQ events that is computationally simple and fairly accurate (Chilukuri et al, 2004; Ibrahim et al, 2000; Ibrahim et al, 2001; Thapar et al, 2003). Fuzzy logic system 
(FLS) has strong inference capabilities of expert system as well as power of natural knowledge representation. The rules of this AI technique are based on modeling human experience and expertise. A membership function provides a measure of the degree of similarity of an element in the fuzzy subset (Mendel, 1995). The basic block diagram of fuzzy logic system is shown in Fig. 3.

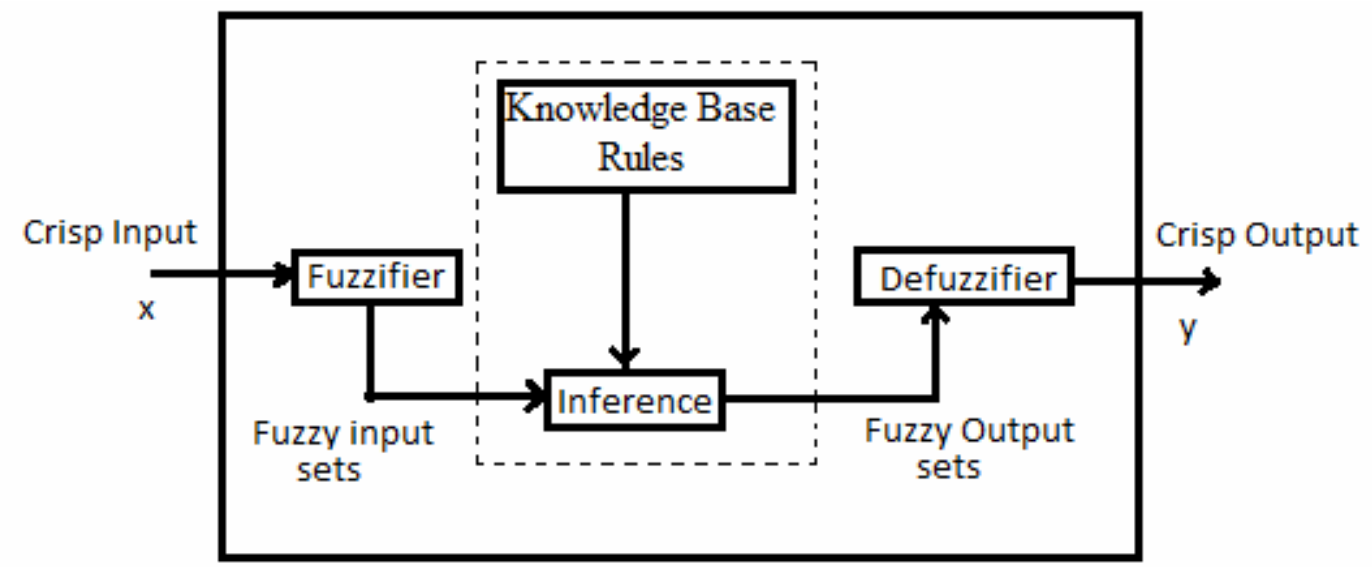

Figure 3. Fuzzy logic system

The fuzzifier maps crisp numbers into fuzzy sets. It is needed in order to activate rules which are in terms of linguistic variables having fuzzy sets associated with them. This step is also called fuzzy matching, which calculates the degree that the input data match the conditions of the fuzzy rules. The inference engine of the FLS maps fuzzy sets into fuzzy sets. It handles the way in which rules are combined. There are two common approaches for the inferences, namely, the clipping method and the scaling method. Both methods generate the conclusion by suppressing the membership function of the consequent. The extent to which they suppress the membership function depends on the degree to which the rule is matched. The clipping method cuts off the top of the membership function, whose value is higher than the matching degree. The scaling method scales down the membership function in proportion to the matching degree.

Knowledge base, which is a set of fuzzy rules, is expressed as a collection of if -then statements which may be provided by the experts. The antecedent (the rule's premise) describes the degree to which input variable matches fuzzy rules and the membership function of the output variable is assigned by (the rule's consequent) the conclusion. In many applications, crisp numbers must be obtained at the output of a FLS. The defuzzifier maps output sets into crisp numbers. Among the various types of defuzzification methods, the center of area (COA, or centroid) and maximum are the two most widely used techniques. The COA derives the crisp number by calculating the weighted average of the output fuzzy set while the maximum membership degree as the crisp number.

Ortiz et al (2006) have proposed a fuzzy expert system for detection and classification of voltage sags. Bizjak et al (2006) proposed an extended fuzzy reasoning scheme for identifying eight kinds of power quality disturbances. The extracted features in wavelet domain and fast Fourier transform (FFT) are used to form the linguistic rules. Simple disturbances such as outage can be detected without the use of DWT and FFT. In (Dash et al, 2000), a hybrid scheme using a Fourier linear combiner and fuzzy expert system has been presented. The captured waveforms have been passed through a Fourier linear combiner block to extract amplitude and phase of the fundamental signal. The proposed method was found to be accurate and robust in presence of noise. It is computationally simple and gives classification result in less than a cycle as compared to the proposed ANN based classifier in (Dash et al, 1996) which produces large error in presence of noise and is computationally intensive.

The main disadvantage of fuzzy classifier is that system time response slows down with the increase in number of rules. If the system does not perform satisfactorily, then the rules are reset again to obtain efficient results i.e. it is not adaptable according to the variation in data. The accuracy of the system is dependent on the knowledge and experience of human experts. The rules should be updated and weighting factors in the fuzzy sets should be refined with time. Neural networks, genetic algorithms, swarm optimization techniques, etc. can be used to for fine tuning of fuzzy logic control systems.

\subsection{Adaptive Neuro-Fuzzy System Based Classifier}

Adaptive neuro-fuzzy system (ANFS) is a hybrid system incorporating the learning abilities of ANN and excellent knowledge representation and inference capabilities of fuzzy logic (Jang, 1993; Jang et al, 1995; Lin et al, 1991) that have the ability to self modify their membership function to achieve a desired performance. An adaptive network, which subsumes almost all kinds of neural network paradigms, can be adopted to interpret the fuzzy inference system. ANFS utilizes the hybrid-learning rule and manage complex decision-making or diagnosis systems. ANFS has proven to be an effective tool for tuning the membership functions of fuzzy inference systems. Reference (Ibrahim et al, 2001) proposed an adaptive neuro fuzzy system to learn power 
quality signature waveform. It is showed that adaptive fuzzy systems are very successful in learning power quality waveform. The new adaptive neuro-fuzzy tool will enhance the performance of the existing power quality service in several ways:

- Maximizing the knowledge gain from the huge amount of power quality data available.

- By setting the proper boundaries of abnormal behavior, unnecessary invocation of more complex PQ analysis software will be prevented, hence the efficiency (including cost effectiveness) of the service currently offered will be increased drastically.

- The adaptive technique used eliminates the need for any prior PQ knowledge on behalf of the customer or system engineers.

\subsection{Support Vector Machines}

Support vector machines (SVMs), which are relatively recent development (Vapnik, 1998; Cristianini et al, 2000; Bishop, 2008), are a set of related supervised learning methods, introduced in the last decade, for pattern recognition and regression, and belong to a family of generalized linear classifiers. In another terms, SVM is a classification and regression prediction tool that uses machine learning theory to maximize predictive accuracy while automatically avoiding over-fit to the data. SVMs are based on minimization of the misclassification probability of unseen patterns with an unknown probability distribution of data and have solid theoretical foundation rooted in statistical learning theory. Real world problems often require hypothesis spaces that are more complex than those using linear discriminants. SVMs are able to find non-linear boundaries if classes are linearly non-separable. The main issue of interest in using SVM for classification is its generalization performance. SVM performs better than neural networks in term of generalization (Cristianini et al, 2000; Bollen et al, 2007; Dwivedi et al, 2008; Bishop, 2008).

Applications within power systems using SVM have been reported in (Moulin et al, 2004; Thukaram et al, 2005; Janik et al, 2006). In (Bishop, 2008), a classifier based on radial basis function (RBF) network and SVM has been proposed and compared for classification of four classes of PQ disturbances. It is claimed that the SVM classifier is particularly effective in the automatic classification of voltage disturbances. The investigation revealed that the SVM network has satisfactory generalization ability and is able to recognize sags and other disturbances correctly, for the wide range of variable parameters. In another work (Axelberg et al, 2007), SVM based algorithm has been proposed for classification of common types of voltage sag disturbances. The results have shown high classification accuracy which implies that, the SVM classification technique is an attractive choice for classification of voltage sag and other PQ disturbances. It has also been found that the accuracy of the proposed method is also dependent on the features given to the classifier. The other advantage of SVM based system is that it is straight forward to extend the system when new types of disturbances are added to the classifier.

Bollen et al (2007) proposed a method based on statistical learning and SVMs for classification of five common types of voltage disturbances. The proposed SVM classifier demonstrated high performance even when training data and test data originate from different networks. Using features from both time domain (RMS signatures) and frequency domain (harmonic magnitudes and total harmonic distortions) effectively characterize the disturbance classes. SVM has originally designed for binary classification and effectively extending it for multiclass classification is still an ongoing research issue (Bunke et al, 2008; Cristianini et al, 2000; Bishop et al, 2008).

\subsection{Summary of PQ Classification Method}

In literature, several methods have been tested for the PQ event classifications. Papers related to various approaches are summarized in Table 2. Reference (Bollen et al, 2007) used the both expert system and support vector approaches for PQ event classification. The strength and weakness of different artificial techniques such as expert system (ES), fuzzy system (FS), neural network (NN), genetic algorithm (GA) and support vector machine (SVM) has been presented in Table 3 (Negnevitsky, 2004).

Table 2: List of references using different approaches

\begin{tabular}{|l|l|}
\hline \multicolumn{1}{|c|}{ Classification Approaches } & \multicolumn{1}{c|}{ References } \\
\hline Artificial Neural Network Based Classifiers & $\begin{array}{l}\text { Perunicic } \text { et al, 1998; Santoso et al, 2000c; Gaouda } \text { et al, 2002a; } \\
\text { Giang, 2004; He } \text { et al, 2006; Mishra et al, 2008. }\end{array}$ \\
\hline Expert System Based Classifier & $\begin{array}{l}\text { Santoso et al, 2000b; Styvaktakis } \text { et al, 2001; Styvaktakis } \text { et al, } \\
\text { 2002; Chung et al, 2002; Reaz et al, 2007. }\end{array}$ \\
\hline Fuzzy Expert System Based Classifiers & $\begin{array}{l}\text { Dash } \text { et al, 2000; Thapar et al, 2003; Zhu et al, 2004; Chilukuri } \text { et } \\
\text { al, 2004; Ortiz } \text { et al, 2006; Bizjak et al, 2006. }\end{array}$ \\
\hline Adaptive Neuro-Fuzzy System Based Classifier & Ibrahim et al, 2001. \\
\hline Support Vector Machines & Moulin et al, 2004; Lin et al, 2006; Axelberg et al, 2007. \\
\hline Artificial Neural Network \& Support Vector Machines & Thukaram et al, 2005; Janik et al, 2006. \\
\hline
\end{tabular}


Table 3: Strength and weakness of different AI techniques

\begin{tabular}{|l|l|l|l|l|l|}
\hline \multirow{2}{*}{ Attributes } & \multicolumn{5}{|c|}{ AI Techniques } \\
\cline { 2 - 6 } & $\begin{array}{l}\text { Expert } \\
\text { System }\end{array}$ & $\begin{array}{l}\text { Fuzzy } \\
\text { System }\end{array}$ & $\begin{array}{l}\text { Neural } \\
\text { network }\end{array}$ & $\begin{array}{l}\text { Genetic } \\
\text { Algorithm }\end{array}$ & $\begin{array}{l}\text { Support Vector } \\
\text { Machine }\end{array}$ \\
\hline Knowledge representation & Rather good & Good & Bad & Rather bad & Bad \\
\hline Uncertainty tolerance & Rather good & Good & Good & Good & Very good \\
\hline Imprecision tolerance & Bad & Good & Good & Good & Very good \\
\hline Adaptability & Bad & Rather bad & Good & Good & Good \\
\hline Learning ability & Bad & Bad & Good & Good & Very good \\
\hline Explanation ability & Good & Good & Bad & Rather bad & Bad \\
\hline Knowledge discovery \& data mining & Bad & Rather bad & Good & Rather good & Good \\
\hline Maintainability & Bad & Rather good & Good & Rather good & Good \\
\hline Generalization Performance & Bad & Bad & Good & Bad & Excellent \\
\hline
\end{tabular}

\section{Classification of Multiple Disturbances}

The majority of classification techniques discussed above is for single disturbances. Incidence of more than one disturbance in an electric system at same time interval is an ordinary situation owing to the presence of several sources of disturbances in power systems. During an abnormal behavior of power system, power line signal may be corrupted by the various disturbances occurring simultaneously i.e. multiple disturbances, and the performance of the above methods may have limited application since they have to deal with multiple disturbances, even though they have not been designed to do so.

In reference (Abdel-Galil et al, 2004; He et al, 2006), classifications of single and two kinds of multiple disturbances have been proposed but further studies are still required. Ribeiro et al (2007) proposed the principle of divide and conquer to decompose electric signal into a set of primitive components for classification of single and multiple disturbances in electric network. The proposed technique is computationally complex and cannot classify flicker, inter harmonics and unbalances. In (Lin et al, 2006), one-versus-one approach based SVM (OSVM) is proposed to classify multiple power quality disturbances. OSVM could detect multiple harmonics and voltage disturbance simultaneously. The above proposal method requires less memory space and processing time by reducing a great quantity of training data. Ferreira et al (2009) proposed (ICA) independent component analysis (Hyvarinen et al, 2001) for analyzing PQ events with multiple disturbances. The method was able to decouple the information between independent disturbance sources for simulated and experimental data.

\section{Effect of Noise on PQ Event Classifiers}

In practice, signals captured by monitoring devices are often accompanied with noise thereby affecting the extraction of important features from the signal. Noise has an adverse effect on the performances of wavelet based event detection, time localization and classification schemes due to the difficulty of separating noise and the disturbances. Very few research works have addressed the effect of electrical noise on performance of wavelet based detection and feature extraction techniques. The disturbance component of the waveform carries the most important information for detection and classification of the disturbances. When a PQ data is decomposed using wavelet transform, most of the disturbance components are reflected at higher frequency bands which are also occupied by noise. Therefore, even if the magnitude of noise level present is not very high compared to fundamental component, for many PQ disturbances, it is comparable to disturbance energy at these bands. Hence, presence of noise degrades the detection capability of wavelet based PQ monitoring system. Electrical noise riding on the PQ waveform data also modulates wavelet domain energy distribution patterns of disturbances which, in turn, degrade performance of the existing classification schemes utilizing wavelets for feature extraction, under practical conditions.

Wavelet based denoising techniques to remove the effect of noise on PQ waveform data (Yang et al, 2000; Elmitwally et al, 2001; Yang et al, 2001; Gaouda et al, 2002b) have been proposed in the literature but, their performance degrades with decrease in the signal to noise ratio (SNR). Among these, (Yang et al, 2001) proposed a promising method for denoising of PQ waveform data to improve the performance of wavelet based PQ monitoring systems. But, there are fundamental problem in the formulation of the proposed technique. Most of the above works generally deal with the denoising of PQ signals from detection and localization point of view (Yang et al, 2000; Gaouda et al, 2002b; Elmitwally et al, 2001). However, the robustness of the energy features, extracted for classification using DWT in the presence of noise and its effect on classification accuracy is rarely addressed.

Dwivedi et al (2006) discussed the effect of noise on the energy features in wavelet domain, extracted for detection and classification of PQ disturbances, and proposed simple and very low complex technique for the extraction of energy features, in noisy environment. The proposed feature extraction method does not require the tedious job of denoising at each and every 
wavelet coefficients of the signal and hence, it is computationally very efficient and is potentially capable in extracting accurately the required energy features even at very low SNR. Two standard statistical hypothesis test based (likelihood ratio (LRT) based and Kolmogorov-Smirnov (KS) goodness-of-fit based) procedure to denoise the PQ waveform have been proposed in (Dwivedi et al, 2009) to enhance the performance of DWT based monitoring system and to avoid miss-alarming. LTR based denoising scheme performs superior to KS test based scheme. Reference (Dwivedi et al, 2010) proposed a low complex technique which exploits the local structure of wavelet coefficients obtained from wavelet multi-resolution decomposition of PQ waveform data as well as high correlation of adjacent wavelet scales for enhancing detection, segmentation and time localization performance of DWT based PQ monitoring systems under practical conditions of harmonics and noise.

\section{Key Issues and Challenges in Classification of PQ Disturbances}

Accurate PQ disturbance classification, which depends on the several factors, is a difficult task. The following are the some of the major issues and challenges in automatic classification of PQ disturbances.

- There exist a few literatures on automatic classification of PQ event based on underlying cause of the disturbance. Most of the methods deal with the type of the PQ event without specifying the underlying cause. For example, for a voltage sag event, it is desirable to know not only voltage sag, but also whether it is caused by the switching of a large load, a line-toground fault, or any other reason. The work should be extended towards cause based classification instead of phenomenon based classification for better understanding of PQ events.

- Classification of swell, dips and interruption i.e. the classification of voltage magnitude events are addressed in many classification algorithms. New algorithms for classification of transients and harmonics distortion have to be explored. Transient identification requiring high frequency waveform recording devices and more robust classification methods is rarely addressed in literature with the exception of (Mamishev et al, 1996).

- Effort should be aimed at incorporating knowledge and expertise of power system engineers in statistical classifiers also.

- The majority of classification techniques proposed is for single disturbances. Therefore, efforts need to be done for multiple disturbance classification. Currently, there are different approaches available for multi-class SVM. These approaches can be tested for their suitability in classification of PQ disturbances.

- Performance of a classifier is highly dependent on the input extracted features. FT, WT and/or model based methods are the optimal starting point for generating features to be proved. The wavelet transform has limited utility in detecting, extracting sag disturbances features because the gradient of the disturbance events are comparable to that of the background signal.

- Choice of a suitable mother wavelet function is another issue of concern in the classifier with the wavelet based extracted features. Because of its compact and localized properties in time frequency plane, Daubechies db4 has been the most frequently used. Another issue of concern is the number of decomposition level required to avoid possible loss of some important information and to have accurate classifier since PQ disturbances cover a wide range of frequency.

- To properly monitor the PQ events, the power quality monitors are installed in the system. Since, it is not possible to install the PQ monitor at all the nodes in the system due to technical and economical reasons, the optimal number of monitoring devices are to be placed in the system to get complete information about the PQ events.

- Most of the studies have done training and testing on synthetic data. Therefore, accumulation of a comprehensive standard PQ database similar to that of many other signal processing fields, for testing and comparisons of the state of the art techniques are also needed.

- Both the economics and the technical limitations must be considered before reaching at solutions. Also, the solutions need to be evaluated using system perspective i.e. possible solutions should be identified at all levels of system from utility supply to the end user's equipment being affected.

- Noise present in the signal has been a major hurdle in the accurate feature extraction and classification of PQ events. Some methods have been proposed to denoise the signal but denoising is still a challenge to power engineers working in the area of PQ analysis and classification.

\section{Conclusion}

Electric power quality, which is a current interest to several power utilities all over the world, is often severely affected by harmonics and transient disturbances. Due to increased use of various power electronic devices in modern power systems, power quality is becoming an important and challenging issue for the power engineers. There is no unique model which can assess the power quality problem and to identify and classify them properly. Existing automatic recognition methods need improvement in terms of their versatility, reliability, and accuracy. The AI tools have been applied for the PQ classification.

This paper addresses different PQ classification approaches in general and AI tools are reviewed in particular. Major key issues and challenges related to these advanced techniques in automatic classification of PQ problems are highlighted. New 
intelligent system technologies using DSP, expert systems, AI and machine learning provide some unique advantages in intelligent classification of PQ distortions.

To build an ideal PQ classification system, various issues have to be addressed.

- The new algorithms should be able to classify voltage or current transients due to different power system events, such as motor starting, transformer inrush currents, capacitor switching, and high impedance faults.

- The new methods need to be capable of implementing cause-based classifications, in addition to phenomenon-based classifications.

- The new methods should classify the multi PQ events occurring simultaneously.

- The new methods should be noise tolerant as PQ signals are normally accompanied with noise.

\section{Acknowledgement}

Dipti Saxena is grateful to the CM and Director, Invertis Institute of Engineering and Technology, Bareilly for permitting her to carry our PhD work from UP Technical University, Lucknow, India.

\section{References}

Abdel-Galil T.K., Kamel M., Youssef A.M., El-Saadany E.F., and Salama M.M.A., 2004. Power quality disturbance classification using the inductive inference approach, IEEE Trans. on Power Delivery, vol. 19, pp. 1812 - 1818.

Acha E. and Madrial M., 2001. Power system harmonics: computer modeling and analysis, London, John Wiley and Sons Ltd.

Agostino Ralph B D', and Stephens Michael A., 1986. Goodness-of-fit techniques, Marcel Dekker Inc., New York, NY.

Allen B. and Rabiner L.R., 1977. A unified approach to short time Fourier analysis and synthesis, IEEE Proc., vol. 65, pp. 15581564.

Altes R., 1980. Detection estimation and classification with spectrograms, Journal of the Acoustical Society of America, vol. 67.

Angrisani L., Daponte P., Apuzzo M.D’ and Testa A., 1998. A measurement method based on the wavelet transform for power quality Analysis, IEEE Trans. on Power Delivery, vol. 13, pp. 990-998.

Arrillaga J., Bollen M.H.J. and Waston N.R., 2000a. Power quality following deregulation, Proc. IEEE., vol. 88, no. 2, pp. 246261.

Arrillaga J., Watson N.R. and Chen.S., 2000b. Power system quality assessment, John Wiley and Sons Ltd., London.

Ashton P.T. and Swift G.W., 1990. Measurements of transient electrical noise on low voltage distribution systems, IEEE Industry Applications Society Annual Meeting, vol. 2, pp. 1740-1742.

Axelberg P.G.V., Gu I.Y.H. and Bollen M.H.J., 2007. Support vector machine for classification of voltage disturbances, IEEE Trans. on Power Delivery, vol. 22, no.3, pp. 1297-1303.

Bishop C.M., 1995. Neural networks for pattern recognition, Oxford University Press, Oxford.

Bishop C.M., 2008. Pattern recognition and machine learning, Springer, New York, USA.

Bizjak Boris. and Planinsic Peter.,2006. Classification of power disturbances using fuzzy logic, Proc. of IEEE Power Electronics and Motion Control Conference, EPE-PEMC 2006, Portoroz, Slovenia.

Bollen M.H.J. and Gu I.Y.H., 2006. Signal processing of power quality disturbances”, IEEE Press, New York, USA.

Bollen M.H.J., Gu I.Y.H., Axelberg P.G.V. and Styvaktakis E., 2007. Classification of underlying causes of power quality disturbances: deterministic versus statistical methods, EURASIP Journal on Advances in Signal Processing, pp.1-17.

Bunke H., and Kandel A., Las M., 2008. Applied pattern recognition, Springer, New York, USA.

Burke J.J., Grifith D.C., and Ward J., 1990. Power quality-Two different perspectives, IEEE Trans. on Power Delivery, vol. 5, pp. 1501-1513.

Chilukuri M.V. and Dash P.K., 2004. Multi-resolution S-transform-based fuzzy recognition system for power quality events, IEEE Trans. on Power Delivery, vol. 19, no. 1, pp. 323 - 330.

Chung J., Powers E.J., Grady W.M., and Bhatt S.C., 2002. Power disturbance classifier using a rule-based method and wavelet packet-based hidden markov model, IEEE Trans. on Power Delivery, vol. 17, pp. 233-241.

Cristianini N. and Shawe-Taylor J., 2000. An introduction to support vector machines, MA: Cambridge Univ. Press, Cambridge.

Dash P.K., Mishra S., Salama M.M.A., and Liew A.C., 2000. Classification of power system disturbances using a fuzzy expert system and a Fourier linear combiner, IEEE Trans. on Power Delivery, vol. 15, pp. 472-477.

Dash P.K., Panigrahi B.K., and Panda G., 2003. Power quality analysis using S-transform, IEEE Trans. on Power Delivery, vol. 18, no. 2, pp. 406-411.

Dash P.K., Swain D.P., Liew A.C. and Raman S., 1996. An adaptive linear combiner for on-line tracking of power system harmonics, IEEE Trans. on Power Systems, vol. 11, no.4, pp.1730-1736.

Domijan A., Heydt G.T., Meliopoulos A.P.S., Venkata S.S., and West S., 1993. Directions of research in electric power quality, IEEE Trans. on Power Delivery, vol. 8, pp. 429-436. 
Dugan Roger.C., McGranaghan M.F., Santoso S. and Beaty H.W., 2003. Electrical power system quality, $2^{\text {nd }}$ Edn., McGraw Hill Book Company, New York.

Dwivedi U.D., Shakya Deepti and Singh S.N., 2008. Power quality monitoring and analysis: an overview and key Issues, International Journal of Systems Signal Control and Engineering Application, Vol., 1, No.1, pp. 74-88.

Dwivedi U. D. and Singh S.N., 2006. A robust energy features estimation for detection and classification of power quality disturbances, Proc. 2006 IEEE PES Power India Conference, New Delhi, pp. 384-390.

Dwivedi U. D., and Singh S.N., 2009. De-noising techniques with change-point approach for wavelet-based power quality monitoring IEEE Trans. on Power Delivery vol. 24, no. 3, pp. 1719-1727.

Dwivedi U.D., and Singh S.N., 2010. Enhanced detection of power quality events using intra and inter-scale dependencies of wavelet coefficients, IEEE Trans. on Power Delivery, vol. 25, no. 1, pp. 358-366.

Elmitwally A., Farghal S., Kandil M., Abdelkader S. and Elkateb M., 2001. Proposed wavelet-neurofuzzy combined system for power quality violations detection and diagnosis, IEE Proc., Generation, Transmission and Distribution, vol. 148, pp. 15 - 20.

Ferreira Danton D., Seixas Jose M. de and Cerqueira Augusto S.,2009. ICA-based method for power quality disturbance analysis, Proc of 15th Intelligent System Application to Power, Curitaba, Brazil.

Gaouda A.M., Kanoun S.H., and Salama M.M.A., 2001. On-line disturbance classification using nearest neighbor rule, Electrical Power System Research, vol. 57, no. 1, pp. 1-8.

Gaouda A.M., Kanoun S.H., Salama M.M.A., and Chikhani A.Y., 2002a. Pattern recognition application for power system disturbance classification, IEEE Trans. on Power Delivery, vol. 17, pp. 677-683.

Gaouda A.M., Kanoun S.H., Salama M.M.A., and Chakhani A.Y., 2002b. Wavelet-based signal processing for disturbance classification and measurement, Proc. IEE, Generation, Transmission and Distribution, vol. 149, pp. 310-318.

Gaouda A.M., Salama M.M.A., Sultan M.R., and Chikhani A.Y., 1999. Power quality detection and classification using waveletmultiresolution signal decomposition, IEEE Trans. on Power Delivery, vol.14, pp. 1469-1476.

Gargoom A.M., Ertugrul N., and Soong W.L., 2007. Investigation of effective automatic recognition systems of power-quality events, IEEE Trans. on Power Delivery, vol. 22, no. 4, pp. 319 - 2326.

Gevarter W.B., 1985. Intelligent machines, an introductory perspective of artificial intelligence and robotics, Prentice-Hall.

Giang Z.L., 2004. Wavelet based network for power disturbance recognition and classification, IEEE Trans. on Power Delivery, vol. 19, no. 4, pp. 1560-1568.

Grenier Y., 1983 Time-dependent ARM modeling of non-stationary signals, IEEE Trans. on Acoustics, Speech, and Signal Processing, vol. 31, no.4, pp. 899-911.

$\mathrm{Gu}$ Y.H., Bollen M.H.J., 2000. Time-frequency and time-scale domain analysis of voltage disturbances survey, IEEE Trans on Power Delivery, vol. 15, pp. 1279-1284.

Hayes-Roth F., Waterman D.A., and Lenat D.B., 1983. Eds., Building expert systems, Addison-Wesley.

He H. and Starzyk J.A., 2006. A self- organizing learning array system for power quality classification based on wavelet transform, IEEE Trans. on Power Delivery, vol. 21, no. 1, pp. 286-295.

Hyvarinen, Karhunen J. and Oja E., 2001. Independent component analysis”, John Wiley \& Sons.

Ibrahim W.R. Anis and Morcos M.M., 2000. Preliminary application of an adaptive fuzzy system for power quality diagnostics, IEEE Power Engineering Review, vol. 20, pp. 55-58.

Ibrahim W.R.A. and Morcos M.M., 2001. An adaptive fuzzy technique for learning power-quality signature waveforms, IEEE Power Engineering Review, vol. 21, no.1, pp. 56-58.

Ibrahim W.R.Anis and Morcos M.M., 2002. Artificial intelligence and advanced mathematical tools for power quality applications: a survey, IEEE Trans. on Power Delivery, vol. 17, pp. 668-673.

Janik P. and Lobos T., 2006. Automated classification of power-quality disturbances using SVM and RBF networks, IEEE Trans. on Power Delivery, vol. 21, no.3, pp. 1663-1669.

Jang J.S.R.,1 993. ANFIS: Adaptive network based fuzzy inference system, IEEE Trans. on Systems, Man and Cybernetics, vol. 23, no. 3, pp. 665-685.

Jang J.S.R. and Sun C.T., 1995. Neuro-fuzzy modeling and control", IEEE Proc., vol. 83, no.3, pp. 378-406.

Kajihara H.H., 1968. Quality power for electronics, Electro-Technology, vol.82, no.5.

Kazibwe W.E. and Sendaula H.M., 1992. Expert system targets power quality issues, IEEE Computer Application in Power, vol. 5, pp. 29-33.

Kezunovic M. and Rikalo I., 1999. Automating the analysis of faults and power quality, IEEE Computer Applications in Power, vol. 12, pp. 46-50.

Lee J.S., Lee C.H., Kim J.O., and Nam S.W., 1997. Classification of power quality disturbances using orthogonal polynomial approximation and bi-spectra, Electronics Letters, vol. 33, no. 18, pp. 1522-1524.

Lin C.T. and Lee C.S.G., 1991. Neural-network-based fuzzy logic control and decision system, IEEE Trans. on Computers, vol. 40, no. 12, pp. 1320-1336.

Lin T., Ying X., and Chen D., 1998. Study on wavelet analysis and its application to numerical protection for large synchronous generator, Proceedings of International Conference on Power System Technology, vol. 2, pp. 1121 -1129. 
Lin Whei-Min, Wu Chien-Hsien, Lin Chia-Hung and Cheng Fu-Sheng., 2006. Classification of multiple power quality disturbances using support vector machine and one-versus-one approach, International Conference on Power System Technology, Chongqing, pp. 1-8.

Mamishev A.V., Short S.X., Kao T.W. and Russel B.D., 1996. Analysis of high impedance faults using fractal techniques, IEEE Trans. on Power Systems, vol. 11, no.1, pp. 435-440.

Martzloff F.D. and Gruzs T.M., 1998. Power quality site surveys: Facts, fiction, and fallacies, IEEE Trans. on Industry Application, vol. 24, pp. 1005-1018.

McGranaghan Mark F. and Santoso Surya, 2007. Challenges and trends in analyses of electric power quality measurement data, EURASIP Journal on Advances in Signal Processing, no. 1, pp. 171-177.

Mendel Jerry M., 1995. Fuzzy logic systems for engineering: a tutorial, IEEE Proc., vol. 83, no. 3,pp. 345-377.

Mishra S., Bhende C.N. and Panigrahi B.K., 2008. Detection and classification of power qulality disturbances using S-transform and probabilistic neural network, IEEE Trans. on Power Delivery, vol. 23, No. 1.

Mo F. and Kinsner W., 1997. Wavelet modeling of transients in power systems, Proc. Of Conference on Communications, Power and Computing, Winnipeg, MB, Canada, pp. 132-137.

Moulin L.S., Silva A.P.A. da., El-Sharkawi M.A., and Marks R.J., 2004. Support vector machines for transient stability analysis of large-scale power systems, IEEE Trans. on Power System, vol. 19, no. 2, pp. 818-825.

Negnevitsky M., 2004. Hybrid intelligent systems: neural expert systems and neuro-fuzzy systems,www.ecst.csuchico.edu/ renner/Teaching/222/Lectures/negnevitsky/lec11_hybrids.pdf, [online].

Ortiz A., Manana M., Ortiz F., C. J., Delgado F.and Eguiluz L. I., 2006. Implementation of a voltage sag characterization based on DSP platform, Proceedings of ICREPQ’ 06, Palma de Mallorca.

Perunicic B., Mallini M., Wang Z., Liu Y., and Heydt G.T., 1998. Power quality disturbance detection and classification using wavelets and artificial neural networks, Proc. 8th Int. Conf. Harmonics and Quality of Power, pp. 77-82.

Reaz M.B.I., Choong F., Sulaiman M. S., Yasin F.M. and Kamada M., 2007. Expert system for power quality disturbance classifier, IEEE Trans. on Power Delivery, vol. 22, no. 3, pp. 1979-1988.

Ribeiro M.V. and Pereira J.L.R., 2007. Classification of single and multiple disturbances in electric signals, EURASIP Journal on Advances in Signal Processing.

Santoso S., Powers E.J., Grady W.M. and Hofmann P., 1996. Power quality assessment via wavelet transform analysis, IEEE Trans. on Power Delivery, vol. 11, pp. 924-930.

Santoso S., Grady W.M., Powers E.J., Lamoree J. and Bhatt S.C., 2000a. Characterization of disturbance power quality event with Fourier and wavelet transforms, IEEE Trans. on Power Delivery, vol. 15, pp.247-254.

Santoso S., Lamoree J., Grady W.M., Powers E.J., and Bhatt S.C., 2000b. A scalable PQ event identification system, IEEE Trans. on Power Delivery, vol. 15, pp. 738-743.

Santoso S., Powers E.J., Grady W.M., and Parsons A.C., 2000c. Power quality disturbance waveform recognition using waveletbased neural classifier- Part1: Theoretical foundation, IEEE Trans. on Power Delivery, vol. 15, pp. 222-228.

Schlabbach, 1994. Expert system measures harmonics and EMC, IEEE Computer Application in Power, vol. 7, pp. 26-29.

Shapiro J.M., 1993. Embedded image coding using zero trees of wavelet coefficients, IEEE Trans. Signal Processing, vol. 41, no. 12, pp. 3445-3462.

Specht D.F., 1990. Probabilistic neural networks, Neural Networks, vol. 3, no. 1, pp. 109-118.

Styvaktakis E., Bollen M.H.J., and Gu I.Y.H., 2001. Expert system for voltage dip classification and analysis, IEEE PES Summer Meeting, vol. 1, pp. 671-676.

Styvaktakis E., Bollen M.H.J., and Gu I.Y.H., 2002. Expert system for classification and analysis of power system events, IEEE Trans. on Power Delivery, vol. 17, pp. 423-428.

Thapar A., Saha T.K., and Dong Z.Y., 2003. A fuzzy logic based recognition technique for power quality categorization, Proceedings of the Australasian Universities Power Engineering Conference, Christchurch, New Zealand, 28 September-1.

Thukaram D., Khincha H.P. and Vijaynarasimha H.P., 2005. Artificial neural network and support vector machine approach or locating faults in radial distribution systems, IEEE Trans. on Power Delivery, vol. 20, no. 2, pp. 710-720.

Vapnik N., 1998 Statistical learning theory, John Wiley and Sons, New York.

Yang Hong-Tzer. and Liao Chiung-Chou., 2000. A correlation-based noise suppression algorithm for power quality monitoring through wavelet transform, International Conference on Power System Technology, 2000. Proceedings Power Conf. 2000, vol. 3, pp. $1311-1316$

Yang Hong-Tzer, and Liao Chiung-Chou, 2001. A de-noising scheme for enhancing wavelet-based power quality monitoring system, IEEE Trans on Power Delivery, vol. 16, no. 3, pp. 353 - 360.

Yen J., and Langari R., 1999. Fuzzy logic: intelligence, control, and information, Englewood Clipps, NJ: Prentice-Hall.

Youssef A.M., Abdel-Galil T.K., El-Saadany E.F., and Salama M.M.A., 2004. Disturbance classification utilizing dynamic time warping classifier”, IEEE Trans. on Power Delivery, vol. 19, no. 1, pp. 272-278.

Zhu T.X., Tso S.K., Lo K.L., 2004. Wavelet-based fuzzy reasoning approach to power-quality disturbance recognition IEEE Trans. on Power Delivery, vol. 19, pp. 1928-1935. 


\section{Biographical notes:}

D. Saxena obtained B. Tech. in Electrical Engineering from KNIT Sultanpur (UP), India and M. Tech. in Process Control from Netaji Subhas Institute of Technology, New Delhi, India in 1999 and 2003, respectively. Presently, she is working as reader in Electrical and Electronics Engineering Department at Invertis Institute of Engg. \& Technology, Bareilly. She is registered as PhD candidate in UP Technical University Lucknow, India. Her research interests are power quality, power electronics, control systems and DSP application in power.

K.S. Verma received his B. Tech. (Hons) in Electrical Engineering and M. Tech. in Electrical Engineering (Power Systems) both from KNIT Sultanpur (India) in 1987 and 1998, respectively. He obtained his Ph.D. degree in Electrical Engineering (Power Systems) from Indian Institute of Technology, Roorkee, Roorkee (India) in 2003. Presently, Prof Verma is Director, KNIT Sultanpur (India). His research interests include FACTS Technology, distributed generation, power system optimization \& control, power quality and AI application in power system.

S. N. Singh received M. Tech. and Ph.D. from Indian Institute of Technology Kanpur, India in 1989 and 1995, respectively. He is a Professor in the Department of Electrical Engineering, Indian Institute of Technology Kanpur, India. Presently he is on leave from IIT Kanpur and working with the Centre for Electric Technology (CET), Technical University of Denmark, Denmark. His research interests include power system restructuring, power system optimization \& control, voltage security and stability analysis, power system planning, and ANN application to power system problems. He is a Fellow of IE (India), Fellow of IETE (India) and senior member of IEEE.

Received December 2009

Accepted March 2010

Final acceptance in revised form March 2010 